\title{
Detection of Lung Cancer using Multilayer Perceptron Neural Network
}

\author{
Type of article: conference abstract
}

\author{
Farzad Mirzakhani* \\ *MSc Student of Medical Informatics, Medical Informatics Department, Faculty of \\ Medicine, Mashhad University of Medical Sciences, Mashhad, Iran \\ Mirzakhanif921@mums.ac.ir
}

\begin{abstract}
Introduction: Lung cancer is the most common cancer in terms of prevalence and mortality. The cancer can be detected once it is reached to a stage that is visible in the CT imaging. Eighty six percent of the patients with lung cancer because they are late understand their disease, surgery has little effect on their improvement. Therefore, the existence of an intelligent system that can detect lung cancer in the early stages is necessary.

Methods: In this study, a lung cancer dataset of UCI database was used. This dataset consists of 32 samples, 57 variables and 3 classes (each class including 10, 9 and 13 samples). The data were normalized within the range 0 to 1 .Then, to increase the detection speed, the dimensions of the data were reduced by using the Principal Components Analysis (PCA). Then, using a multilayer perceptron neural network, a model for classification and prediction of lung cancer was developed. Finally, the performance of the model was measured using sensitivity, specificity, positive predictive value and negative predictive value. It should be noted that all analyzes were done using Weka software.

Results: After developing and evaluating an artificial neural network model, the developed model had a sensitivity of $66.7 \%$, a $98.5 \%$ specificity, a positive predictive value of $75 \%$, and a negative predictive value of $97.7 \%$.

Conclusion: In intelligent diagnostic systems, in addition to high accuracy of diagnosis, the speed of diagnosis and decision making is also important. Therefore, researchers increased the speed of the prediction model by reducing 57 variables to 8 variables using PCA. Also, the high sensitivity and high specificity of developed model demonstrates high power of artificial neural network model in detecting lung cancer.
\end{abstract}

Keywords: Lung cancer, Artificial Intelligence, Artificial neural network (ANN), Principal component analysis (PCA)

\section{Declaration of conflicts}

This abstract is selected from the First International Congress of Diseases and Health Outcomes Registry and First National Congress of Medical Informatics, 14-17 February 2017, Mashhad, Iran

\section{Authors' biography}

No biography.

\section{References}

No references. 